\title{
Estimation of Parameters of Signal Mixture with Noise Based on Monitoring Outputs of the Multichannel Device of Orthogonal Signals Distinction at Coherent Reception
}

\author{
Sergey B. Zhironkina, Alexander A. Bliznyuk ${ }^{a}$, \\ Andrey A. Pshenitsyn ${ }^{\mathrm{a}}$ and Igor V. Lyutikov*b \\ ${ }^{a}$ Military Academy of Aero-Space Defence \\ named after the Marshal of Soviet Union G.K. Zhukov \\ Tver, Russian Federation \\ ${ }^{b}$ Siberian Federal University \\ Krasnoyarsk, Russian Federation
}

Received 01.09.2019, received in revised form 17.11.2019, accepted 21.01.2020

\begin{abstract}
Mathematical expressions for optimal and quasi-optimal estimations of signal/noise relation and spectral density of noise capacity, based on monitoring outputs of the coherent multichannel device of distinction of orthogonal signals have been received. It was found that, by means of processing outputs of channels in multichannel devices of distinction of orthogonal signals, one can make almost implementable quasi-optimal estimations of signal/noise relation or noise parameters with rather high accuracy.
\end{abstract}

Keywords: signal/noise relation, orthogonal signals, spectral density of noise capacity, optimal coherent reception.

Citation: Zhironkin S.B., Bliznyuk A.A., Pshenitsyn A.A., Lyutikov I.V. Estimation of parameters of signal mixture with noise based on monitoring outputs of the multichannel device of orthogonal signals distinction at coherent reception, J. Sib. Fed. Univ. Eng. \& Technol., 2020, 13(3), 361-369. DOI: 10.17516/1999-494X-0227

(C) Siberian Federal University. All rights reserved

This work is licensed under a Creative Commons Attribution-Non Commercial 4.0 International License (CC BY-NC 4.0).

* Corresponding author E-mail address: lyutikovigor@mail.ru 


\title{
Оценка параметров смеси сигнала с шумом
}

\author{
по наблюдению выходов многоканального \\ устройства различения ортогональных сигналов \\ при когерентном приеме
}

\author{
С.Б. Жиронкин ${ }^{\mathrm{a}}$, А.А. Близнюк ${ }^{\mathrm{a}}$, \\ А.А. Пшеницын ${ }^{\text {a }}$ И.В. Лютиков \\ ${ }^{a}$ Военная академия воздушно-космической оборонь \\ им. Маршала Советского Союза Г.К. Жукова \\ Российская Федераиия, Тверь \\ ${ }^{6}$ Сибирский федеральный университет \\ Российская Федераиия, Красноярск
}

\begin{abstract}
Аннотация. Получены математические выражения для оптимальных и квазиоптимальных оценок отношения сигнал/шум и спектральной плотности мощности шума, формируемых по наблюдению выходов когерентного многоканального устройства различения ортогональных сигналов. Установлено, что, обрабатывая выходы каналов в многоканальных устройствах различения ортогональных сигналов, можно формировать практически реализуемые квазиоптимальные оценки отношения сигнал/шум или параметров шума с достаточно высокой точностью.
\end{abstract}

Ключевые слова: отношение сигнал/шум, ортогональные сигналы, спектральная плотность мощности шума, оптимальный когерентный прием.

Цитирование: Жиронкин, С.Б. Оценка параметров смеси сигнала с шумом по наблюдению выходов многоканального устройства различения ортогональных сигналов при когерентном приеме / С.Б. Жиронкин, А.А. Близнюк, А.А. Пшеницын, И.В. Лютиков // Журн. Сиб. федер. ун-та. Техника и технологии, 2020. 13(3). С. 361-369. DOI: $10.17516 / 1999-494 X-0227$

\section{Введение}

Оценки отношения сигнал/шум и (или) параметров шума необходимы для эффективной работы многих алгоритмов мягкого декодирования, управления мощностью и выделения ресурсов, реализуемых в телекоммуникационных системах [1]. Особую актуальность подобные задачи имеют для каналов с импульсными шумовыми помехами, когда сигнально-помеховая обстановка нестационарна: импульсы (символы, элементы сигнально-кодовой конструкции) принимаются на фоне шума с изменяющимися параметрами [2].

Обычно оценки отношения сигнал/шум и (или) параметров шума формируются в результате обработки последовательных временных отсчетов смеси сигнала с шумом [1], при этом могут использоваться специальные измерительные каналы [3]. В многоканальных устройствах различения ортогональных сигналов необходимую входную статистику (наблюдение) можно снять в один момент времени с выходов каналов и оценить отношение сигнал/шум и (или) параметры шума без использования специальных измерительных каналов.

Цель работы - получение математических выражений для оптимальных и квазиоптимальных оценок отношения сигнал/шум и спектральной плотности мощности шума, формируемых по наблюдению выходов когерентного многоканального устройства различения ортогональ- 
ных сигналов, определение точности оценок методом компьютерного имитационного статистического моделирования.

\section{Оценка отношения сигнал/шум}

При оптимальном когерентном приеме в каждом канале устройства различения сигналов находится коррелятор или согласованный фильтр, на их выходах формируются корреляционные интегралы, которые можно представить в виде

$$
q_{i}=\frac{2}{N} \int_{o}^{T} \xi(t) s_{i}(t) d t
$$

где $\xi(t)=s_{j}(t)+n(t)$ - смесь принимаемого сигнала с белым гауссовским шумом с односторонней спектральной плотностью $N$ [4].

Величины $q_{i}$ имеют гауссовское распределение. При различении ортогональных сигналов $q_{i}$ статистически независимы, их математическое ожидание равно нулю при $i \neq j$, а при $i=j-$ отношению сигнал/шум $Q=\frac{2 E}{N}$, где $E-$ энергия сигнала. Дисперсия $q_{i}$ также равна $Q$.

Обрабатывая совокупность $q_{i}, i=\overline{1, M}, M$ - количество каналов в устройстве различения (количество различаемых сигналов), можно получить оценку $Q$, не выделяя для этого специальный измерительный канал.

Известно решение классической задачи оценки по максимуму правдоподобия математического ожидания и дисперсии гауссовской случайной величины по независимой выборке [5]. Особенностью рассматриваемой задачи является то, что математическое ожидание и дисперсия равны друг другу (функция правдоподобия зависит не от двух, а от одного параметра $Q$ ), причем математическое ожидание отлично от нуля только в одном канале, а номер этого канала неизвестен (оценивается в процессе решения задачи различения).

С учетом перечисленных особенностей наблюдение в рассматриваемой задаче может быть представлено в следующем виде:

$$
q_{i}=\delta_{i j} Q+\sqrt{Q} \cdot n_{i} ; i=\overline{1, M},
$$

где $\delta_{i j}$ - символ Кронекера; $i$ - номер канала в устройстве различения; $j$ - номер принимаемого сигнала, оцениваемый в процессе решения задачи различения; $n_{i}-$ независимые стандартные (с нулевым математическим ожиданием и единичной дисперсией) гауссовские случайные величины.

Функция правдоподобия неизвестных величин $Q$ и $j$ равна

$$
p\left(q_{1}, q_{2}, \ldots, q_{M} \mid Q, j\right)=\frac{1}{\sqrt{2 \pi Q}} \exp \left(-\frac{\left(q_{j}-Q\right)^{2}}{2 Q}\right) \prod_{i=1(i \neq j)}^{M} \frac{1}{\sqrt{2 \pi Q}} \exp \left(-\frac{q_{i}^{2}}{2 Q}\right)
$$

Функцию правдоподобия интересующего нас параметра $Q$ находят из (1) известным способом: 


$$
L(Q)=p\left(q_{1}, q_{2}, \ldots, q_{M} \mid Q\right)=\sum_{j=1}^{M} p\left(q_{1}, q_{2}, \ldots, q_{M} \mid Q, j\right) P(j) .
$$

Применяя этот способ, получим

$$
L(Q)=p\left(q_{1}, q_{2}, \ldots, q_{M} \mid Q, \hat{j}\right)\left(1-P_{e}(Q)\right)+\frac{P_{e}(Q)}{M-1} \sum_{j=1(j \neq \hat{j})}^{M} p\left(q_{1}, q_{2}, \ldots, q_{M} \mid Q, j\right),
$$

где $\hat{j}$ - оценка номера принимаемого сигнала (результат решения задачи различения); $P_{e}(Q)-$ вероятность ошибки при различении сигналов.

При когерентном приеме [4]

$$
P_{e}(Q)=1-\frac{1}{\sqrt{2 \pi}} \int_{-\infty}^{\infty} \exp \left[-\frac{1}{2}(x-\sqrt{Q})^{2}\right] \Phi^{M-1}(x) d x
$$

где $\Phi(x)=\frac{1}{\sqrt{2 \pi}} \int_{-\infty}^{x} \exp \left(-\frac{t^{2}}{2}\right) d t-$ интеграл вероятности.

Уравнение, определяющее оптимальную по критерию максимума правдоподобия оценку $Q$, имеет вид

$$
\frac{d}{d Q} \ln L(Q)=0
$$

Проще найти выражение для квазиоптимальной оценки при малых значениях $P(Q)<<1$. В этом случае $L(Q) \approx p\left(q_{1}, q_{2}, \ldots, q_{M} \mid Q, \hat{j}\right)$ при

$$
p\left(q_{1}, q_{2}, \ldots, q_{M} \mid Q, \hat{j}\right)=\frac{1}{\sqrt{2 \pi Q}} \exp \left(-\frac{\left(q_{\hat{j}}-Q\right)^{2}}{2 Q}\right) \prod_{i=1(i \neq \hat{j})}^{M} \frac{1}{\sqrt{2 \pi Q}} \exp \left(-\frac{q_{i}^{2}}{2 Q}\right) .
$$

Квазиоптимальную оценку $Q$ находим из уравнения

$$
\frac{d}{d Q} \ln p\left(q_{1}, q_{2}, \ldots, q_{M} \mid Q, \hat{j}\right)=0 .
$$

После логарифмирования и взятия производной уравнение принимает вид

$$
-\frac{M}{2 Q}+\frac{1}{2 Q^{2}} \sum_{i=1(i \neq \hat{j})}^{M} q_{i}^{2}+\frac{1}{2 Q^{2}}\left(q_{\hat{j}}-Q\right)^{2}+\frac{1}{Q}\left(q_{\hat{j}}-Q\right)=0 .
$$

В результате дальнейших преобразований приходим к приведенному квадратному уравнению

$$
Q^{2}+M Q-\sum_{i=1}^{M} q_{i}^{2}=0
$$

Корнями этого уравнения являются

$$
\hat{Q}_{1,2}=-\frac{M}{2} \pm \sqrt{\frac{M^{2}}{4}+\sum_{i=1}^{M} q_{i}^{2}} .
$$

Физический смысл имеет только первый корень, определяющий квазиоптимальную оценку отношения сигнал/шум: 


$$
\hat{Q}=-\frac{M}{2}+\sqrt{\frac{M^{2}}{4}+\sum_{i=1}^{M} q_{i}^{2}} .
$$

В соответствии с полученным выражением алгоритм формирования квазиоптимальной оценки инвариантен относительно результата решения задачи различения $\hat{j}$ : выходы $\hat{q}_{i}$ всех каналов используют равнозначно, $\hat{q}_{\hat{j}}$ среди остальных выходов не выделяют.

\section{Оценка спектральной плотности мощности шума}

При неизвестной спектральной плотности шума корреляционные интегралы, формируемые в процессе оптимального когерентного приема, представляются следующим образом:

$$
q_{i}=\int_{o}^{T} \xi(t) s_{i}(t) d t
$$

Величины $q_{i}$ имеют гауссовское распределение. При различении ортогональных сигналов величины $q_{i}$ статистически независимы, их математическое ожидание равно нулю при $i \neq j$, а при $i=j$ - энергии сигнала $E$ (предполагается, что она известна). Дисперсия $q$ равна $\frac{N E}{2}[4]$.

Наблюдение в рассматриваемой задаче может быть представлено в следующем виде:

$$
q_{i}=\delta_{i j} E+\sqrt{\frac{N E}{2}} \cdot n_{i} ; i=\overline{1, M},
$$

где $\delta_{i j}$ - символ Кронекера; $i$ - номер канала в устройстве различения; $j$ - номер принимаемого сигнала, оцениваемый в процессе решения задачи различения; $n$ - независимые стандартные гауссовские случайные величины.

Функция правдоподобия неизвестных величин $N$ и $j$ равна

$$
p\left(q_{1}, q_{2}, \ldots, q_{M} \mid N, j\right)=\frac{1}{\sqrt{\pi N E}} \exp \left(-\frac{\left(q_{j}-E\right)^{2}}{N E}\right) \prod_{i=1(i \neq j)}^{M} \frac{1}{\sqrt{\pi N E}} \exp \left(-\frac{q_{i}^{2}}{N E}\right)
$$

а функция правдоподобия интересующего нас параметра $N$ -

$$
\begin{aligned}
& L(N)=p\left(q_{1}, q_{2}, \ldots, q_{M} \mid N\right)=\sum_{j=1}^{M} p\left(q_{1}, q_{2}, \ldots, q_{M} \mid N, j\right) P(j)= \\
& =p\left(q_{1}, q_{2}, \ldots, q_{M} \mid N, \hat{j}\right)\left(1-P_{e}(N)\right)+\frac{P_{e}(N)}{M-1} \sum_{j=1(j \neq \hat{j})}^{M} p\left(q_{1}, q_{2}, \ldots, q_{M} \mid N, j\right),
\end{aligned}
$$

где $P_{e}(N)$ - вероятность ошибки при когерентном различении сигналов:

$$
P_{e}(N)=1-\frac{1}{\sqrt{2 \pi}} \int_{-\infty}^{\infty} \exp \left[-\frac{1}{2}\left(x-\sqrt{\frac{2 E}{N}}\right)^{2}\right] \Phi^{M-1}(x) d x .
$$

Уравнение, определяющее оптимальную по критерию максимума правдоподобия оценку $N$, имеет вид

$$
\frac{d}{d N} \ln L(N)=0
$$


Выражение для квазиоптимальной оценки получим, как и в предыдущей задаче оценивания отношения сигнал/шум, в предположении о малой величине $P_{e}(N)$. В этом случае $L(N) \approx p\left(q_{1}, q_{2}, \ldots, q_{M} \mid N, \hat{j}\right)$ при

$$
p\left(q_{1}, q_{2}, \ldots, q_{M} \mid N, \hat{j}\right)=\frac{1}{\sqrt{\pi N E}} \exp \left(-\frac{\left(q_{\hat{j}}-E\right)^{2}}{N E}\right) \prod_{i=1(i \neq \hat{j})}^{M} \frac{1}{\sqrt{\pi N E}} \exp \left(-\frac{q_{i}^{2}}{N E}\right) .
$$

Квазиоптимальную оценку $N$ находим из уравнения

$$
\frac{d}{d N} \ln p\left(q_{1}, q_{2}, \ldots, q_{M} \mid N, \hat{j}\right)=0 .
$$

Решение уравнения имеет вид

$$
\hat{N}=\frac{2\left(E-2 q_{\hat{j}}+\frac{1}{E} \sum_{i=1}^{M} q_{i}^{2}\right)}{M} .
$$

Такое же выражение можно получить, воспользовавшись известным результатом решения классической задачи оценки по максимуму правдоподобия дисперсии $D$ гауссовской случайной величины по независимой выборке [5] с учетом того, что выход одного из каналов (предполагаем с номером $\hat{j}$ ) имеет отличное от нуля математическое ожидание, равное Е:

$$
\hat{N}=\frac{2}{E} \hat{D}=\frac{2}{E} \frac{\left(q_{\hat{j}}-E\right)^{2}+\sum_{i=1(i \neq j)}^{M} q_{i}^{2}}{M} .
$$

Отсюда легко выводим (3).

В соответствии с (3) алгоритм формирования квазиоптимальной оценки спектральной плотности шума зависит от результата решения задачи различения $\hat{j}$ : помимо суммы выходов $q_{i}$ всех каналов отдельно используют $q_{\hat{j}}$.

\section{Результаты компьютерного моделирования}

Точность алгоритмов формирования квазиоптимальных оценок отношения сигнал/шум и спектральной плотности шума была определена по результатам компьютерного имитационного статистического моделирования, представленного в виде графиков на рис. 1-3. По вертикальной оси на графиках нормированное (к истинному значению оцениваемого параметра) значение среднеквадратической ошибки.

На графиках рис. 1-3 видно, что, обрабатывая выходы каналов в многоканальных устройствах различения ортогональных сигналов, можно формировать практически реализуемые квазиоптимальные оценки отношения сигнал/шум или параметров шума с достаточно высокой точностью (при оценке спектральной плотности мощности шума, не зависящей ни от отношения сигнал/шум, ни от самой спектральной плотности). При увеличении объема ансамбля ортогональных сигналов точность оценок параметров смеси сигнала с шумом повышается: при $M \geq 128$ среднеквадратическая ошибка снижается ниже уровня 10-15\% от истинного значения параметра. 


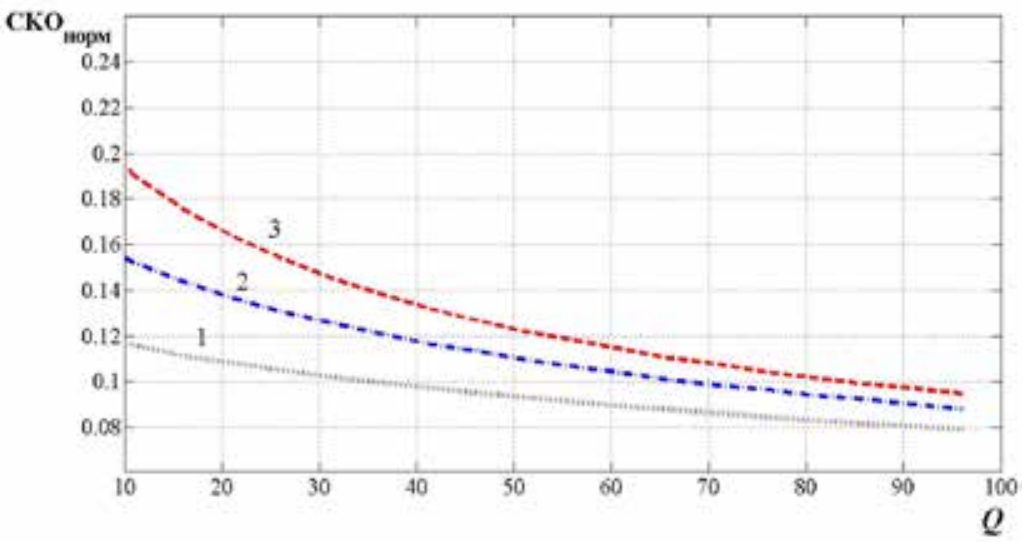

Рис. 1. Зависимость нормированного значения среднеквадратической ошибки оценки отношения сигнал/ шум от его истинного значения: 1 - для $M=128 ; 2$ - для $M=64 ; 3$ - для $M=32$

Fig. 1. Dependence of normirovanny value of a srednekvadratichesky error of an assessment of the relation signal/noise from its true value: 1 - for $M=128 ; 2$ - for $M=64 ; 3$ - for $M=32$

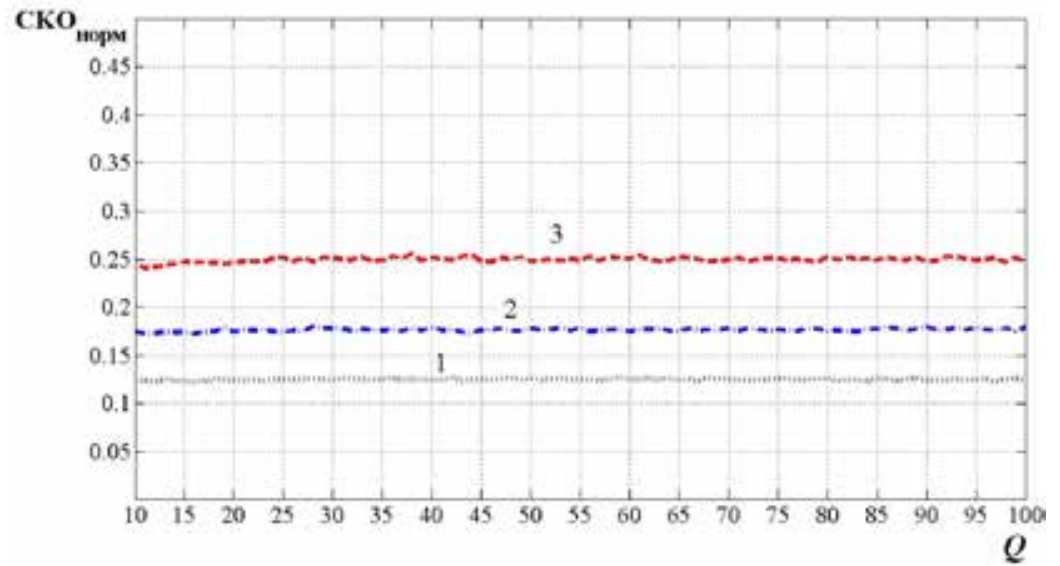

Рис. 2. Зависимость нормированного значения среднеквадратической ошибки оценки спектральной плотности шума от отношения сигнал/шум при фиксированном значении энергии сигнала: 1 - для $M=128 ; 2$ - для $M=64 ; 3$ - для $M=32$

Fig. 2. Dependence of normirovanny value of a srednekvadratichesky error of an assessment of spectral density of noise on the relation signal/noise at the fixed value of energy of a signal: 1 - for $M=128 ; 2$ - for $M=64 ; 3$ - for $M=32$ 


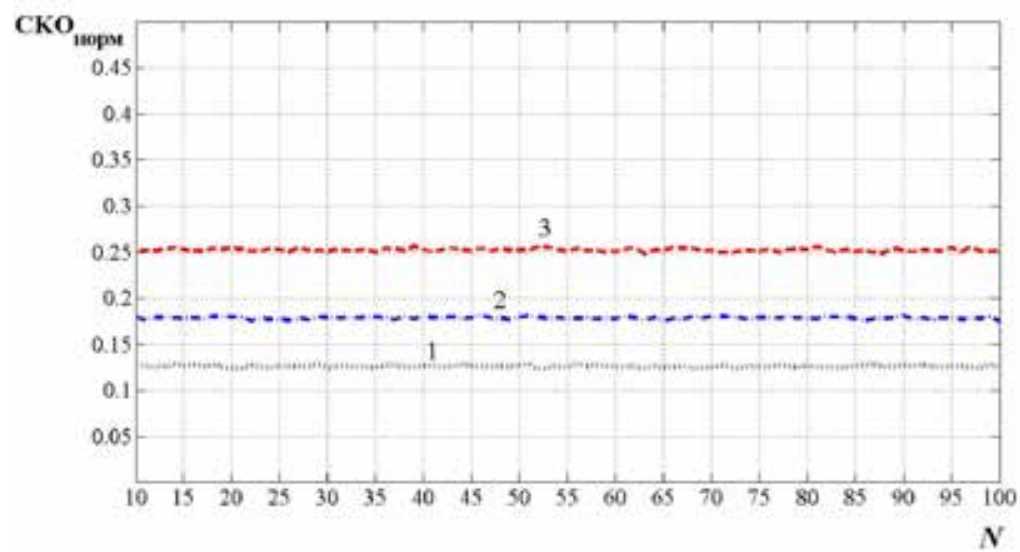

Рис. 3. Зависимость нормированного значения среднеквадратической ошибки оценки спектральной плотности шума от ее истинного значения при $Q=10: 1$ - для $M=128 ; 2$ - для $M=64 ; 3$ - для $M=32$

Fig. 3. Dependence of normirovanny value of a srednekvadratichesky error of an assessment of spectral density of noise on its true value at $Q=10: 1-$ for $M=128 ; 2$ - for $M=64 ; 3-$ for $M=32$

\section{Заключение}

Таким образом, получены математические выражения для оптимальных и квазиоптимальных оценок отношения сигнал/шум и спектральной плотности мощности шума, формируемых по наблюдению выходов когерентного многоканального устройства различения ортогональных сигналов. Установлено, что, обрабатывая выходы каналов в многоканальных устройствах различения ортогональных сигналов, можно формировать практически реализуемые квазиоптимальные оценки отношения сигнал/шум или параметров шума с достаточно высокой точностью. Эти оценки могут быть использованы для эффективной работы многих алгоритмов мягкого декодирования, управления мощностью и выделения ресурсов, реализуемых в телекоммуникационных системах. При увеличении объема ансамбля ортогональных сигналов точность оценки параметров смеси сигнала с шумом повышается, поэтому помехоустойчивость мягкого декодирования также должна повышаться. Это является дополнительным (к известным положениям $[3,4])$ обоснованием целесообразности увеличения объема ансамбля ортогональных сигналов.

\section{Список литературы / References}

[1] Мелентьев О.Г., Шевнина И.Е. Модификация алгоритма оценки отношения сигнал/ шум. Вестник СибГУТИ, 2018, 2, 3-7 [Melentyev O.G., Shevnina I.E. Updating of algorithm of an assessment of the relation signal/noise, Vestnik SibGUTI, 2018, 2, 3-7 (in Russian)].

[2] Жиронкин С.Б., Близнюк А.А., Кучин А.А. Помехоустойчивость запросного канала опознавания с широкополосными сигналами и корректирующими кодами в условиях импульсных шумовых и внутрисистемных помех. Журнал Сибирского федерального университета. Техника и технологии, 2019, 12 (6), 673-682 [Zhironkin S.B., Bliznuk A.A., Kuchin A.A. A noise stability of the request channel of an identification with broadband signals and correcting codes in the conditions of pulse noise and intersystem hindrances, Journal 
of Siberian Federal University. Engineering and technologies, 2019, 12 (6), 673-682 (in Russian)].

[3] Варакин Л.Е. Системы связи с шумоподобными сигналами. М.: Радио и связь, 1985, 384 c. [Varakin L.E. Communication systems with noise-type signals. M.: Radio i svyaz, 1985, 384 p. (in Russian)].

[4] Тихонов В.И. Оптимальный прием сигналов. М.: Радио и связь, 1983, 320 с. [Tikhonov V.I. Optimum reception of signals. M.: Radio i svyaz, 1983, 320 p. (in Russian)].

[5] Тихонов В.И. Статистическая радиотехника. М.: Радио и связь, 1982. 624 с. [Tikhonov V.I. Statistical radio engineering. M.: Radio i svyaz, 1982, 624 p. (in Russian)]. 